\title{
Tuberculosis Is Not a Risk Factor for Primary Biliary Cirrhosis: A Review of the Literature
}

\author{
Daniel S. Smyk, ${ }^{1}$ Dimitrios P. Bogdanos, ${ }^{1,2,3}$ Albert Pares, ${ }^{4}$ Christos Liaskos, ${ }^{2}$ \\ Charalambos Billinis, ${ }^{5}$ Andrew K. Burroughs, ${ }^{6}$ and Eirini I. Rigopoulou ${ }^{3}$ \\ ${ }^{1}$ Institute of Liver Studies, King's College Hospital and Division of Transplantation Immunology and Mucosal Biology, \\ School of Medicine, King's College London, London SE5 9RS, UK \\ ${ }^{2}$ Cellular Immunotherapy and Molecular Immunodiagnostics, Center for Research and Technology Thessaly, 41222 Larissa, Greece \\ ${ }^{3}$ Department of Medicine, University Hospital of Larissa, University of Thessaly School of Medicine, 41110 Larissa, Greece \\ ${ }^{4}$ Liver Unit, Hospital Clínic de Barcelona, IDIBAPS, CIBERehd, University of Barcelona, 08036 Barcelona, Spain \\ ${ }^{5}$ Faculty of Veterinary Science, University of Thessaly, 43100 Karditsa, Greece \\ ${ }^{6}$ The Royal Free Sheila Sherlock Liver Centre, Royal Free Hospital and Department of Surgery, University Collegue London, \\ London NW32QG, UK
}

Correspondence should be addressed to Dimitrios P. Bogdanos, dimitrios.bogdanos@kcl.ac.uk

Received 20 July 2012; Accepted 3 October 2012

Academic Editor: Juraj Ivanyi

Copyright (c) 2012 Daniel S. Smyk et al. This is an open access article distributed under the Creative Commons Attribution License, which permits unrestricted use, distribution, and reproduction in any medium, provided the original work is properly cited.

Primary biliary cirrhosis (PBC) is a progressive cholestatic liver disease characterised serologically by cholestasis and the presence of high-titre antimitochondrial antibodies, and histologically by chronic nonsuppurative cholangitis and granulomata. As PBC is a granulomatous disease and Mycobacterium tuberculosis is the most frequent cause of granulomata, a causal relation between tuberculosis and $\mathrm{PBC}$ has been suggested. Attempts to find serological evidence of PBC-specific autoantibodies such as AMA have been made and, conversely, granulomatous livers from patients with PBC have been investigated for molecular evidence of Mycobacterium tuberculosis. This paper discusses in detail the reported data in support or against an association between Mycobacterium tuberculosis infection and PBC. We discuss the immunological and microbiological data exploring the association of $\mathrm{PBC}$ with exposure to Mycobacterium tuberculosis. We also discuss the findings of large epidemiologic studies investigating the association of $\mathrm{PBC}$ with preexistent or concomitant disorders and the relevance of these findings with tuberculosis. Genome-wide association studies in patients with tuberculosis as well as in patients with PBC provide conclusive hints regarding the assumed association between exposure to this mycobacterium and the induction of PBC. Analysis of these data suggest that Mycobacterium tuberculosis is an unlikely infectious trigger of $\mathrm{PBC}$.

\section{Introduction}

Primary biliary cirrhosis (PBC) is a chronic cholestatic, autoimmune liver disease characterised by progressive inflammatory destruction of the small and medium intrahepatic bile ducts and subsequent fibrosis, cirrhosis [1-5], and eventually liver failure $[6,7]$. The disease predominantly affects middle-aged women and is practically absent in children or youngsters [8-11]. PBC affects more than one member within the same family, and several reports indicate that first degree relatives of $\mathrm{PBC}$ patients have an increased risk of developing the disease $[12,13]$. The prevalence of the disease varies among countries, with a recent systematic review indicating prevalence to be $1.91-40.2$ per 100,000 inhabitants, and the incidence to be $0.33-5.8$ per 100,000 inhabitants/year, in European and North American cohorts (although a breakdown of ethnicity was not provided) [14]. There is a consensus, however, that despite the heterogeneity in the estimated prevalence and incidence amongst ethnic groups, the incidence and prevalence of $\mathrm{PBC}$ is increasing [14-18]. The reasons for this increase are poorly understood. Whether there is a true increase or it is due to the awareness 
for the disease amongst clinicians and the meticulous diagnostic assessment, such a disease-specific autoantibody testing remains to be seen.

Several autoantibody profiles have been found to be specific for the disease [19] and aid in the diagnostic workup of PBC. These include antimitochondrial antibodies (AMA) [20-24] and/or disease-specific antinuclear antibody (ANA) [25-27], which are found in both symptomatic and asymptomatic patients [28]. Most common symptoms at presentation are nonspecific and include fatigue, pruritus, Sicca symptomatology, and arthralgias. Autoimmune rheumatic diseases such as Sjögren's syndrome and systemic sclerosis, as well as other extrahepatic autoimmune manifestations such as autoimmune thyroiditis frequently coexist with PBC $[2,29,30]$. In more severe cases, symptoms relate to portal hypertension and hepatic decompensation (jaundice, ascites, or variceal bleeding), which may indicate the need for liver transplantation $[2,5,31]$. The progression of $\mathrm{PBC}$ is generally slow in the majority of the cases $[6$, 31].

The widely accepted diagnostic criteria of PBC consists of three components: (1) biochemical evidence of cholestasis in the form of elevated levels of alkaline phosphatase (ALP) and $\gamma \mathrm{GT},(2)$ seropositivity for disease-specific antimitochondrial antibodies, and (3) histological features on liver biopsy compatible with or diagnostic of PBC [2]. The diagnosis of $\mathrm{PBC}$ is probable when at least two of these criteria are met $[2,4]$. Most cases have elevated levels of immunoglobulin $\mathrm{M}$ (IgM) $[2,4,32]$.

The diagnostic hallmark of $\mathrm{PBC}$ is the presence of hightitre AMA mainly targeting the E2 subunits of the oxo-acid dehydrogenase complexes (OADC), and in particular that of pyruvate dehydrogenase complex (PDC-E2) [21, 24, 28, 33]. Only $3-10 \%$ of patients with PBC lack these antibodies and are considered true AMA-negative PBC patients [28, 34-37]. These autoantibodies are practically nonexistent in patients with other liver diseases unrelated to $\mathrm{PBC}[38,39]$. Also, true AMA seropositivity in patients with autoimmune rheumatic diseases indicates the current or future development of PBC $[23,40]$. Prospective studies have shown that the presence of AMA predicts the future development of $\mathrm{PBC}$ in asymptomatic, cholestatic, or acholestatic individuals $[22,41]$. ANA specific for the disease can also be present in approximately $50 \%$ of patients [42-44]. Published data support the notion that $\mathrm{PBC}$-specific ANA reactivities may have prognostic significance [27, 44-47]. Various other autoantibody specificities have been reported in patients with PBC [48-51].

The most prominent histological features of $\mathrm{PBC}$ include chronic nonsuppurative cholangitis with or without granulomata demonstrating destruction of biliary epithelial cells and loss of small bile ducts with portal inflammatory cell infiltration (see below) $[2,4,5]$. Genetic factors and environmental triggers have been considered important for the induction of autoimmune disease, such as PBC $[8,52-$ 57]. Several infectious and noninfectious triggers have been implicated in the pathogenesis of PBC $[8,12,52,55,58-64]$.

Because Mycobacterium tuberculosis (M. tuberculosis) [65] is the most frequent cause of granulomas [66], and in view of reports indicating serological evidence of $\mathrm{PBC}$ specific AMA in patients with tuberculosis, it has been suggested that tuberculosis/M. tuberculosis may be a cause of PBC (Table 1). Indeed, M. tuberculosis has been indicated as a potential cause of other autoimmune disease such as systemic lupus erythematosus (SLE) [67-74]. This paper will critically analyse the epidemiological, clinical, immunological, and experimental data in support or against the notion that tuberculosis and PBC may be pathogenetically linked.

\section{Granulomas in PBC}

Up to $15 \%$ of liver biopsy material contains evidence of granulomas [82-86]. An analysis of 12,161 liver biopsies revealed the presence of granulomas in 442 (3.6\%) [87] cases, including 215 diagnosed with PBC, representing $48.7 \%$ of all biopsies with granulomas and $1.8 \%$ of all biopsies analysed [87]. Molecular evidence of infectious agents by PCR was found in just 15 samples (3.4\%) and M. tuberculosis has been detected in three of the $15(20 \%)$ but it is not clear whether the M. tuberculosis-positive cases were from livers of patients with $\mathrm{PBC}$ [87]. The fact that three or less of the 215 granulomatous livers from $\mathrm{PBC}$ cases had molecular evidence of $M$. tuberculosis argues against the notion that this mycobacterium is a trigger for the development of this enigmatic disease. Other studies have also noted and reported a diagnosis of PBC in $24-62 \%$ of livers with evidence of granulomas $[82,83,88]$. However, only a small proportion of the PBC granulomata had evidence of mycobacteria, again pointing towards the lack of an association of M. tuberculosis with PBC.

Irrespective of the relation of $\mathrm{PBC}$ granulomas with M. tuberculosis infections, attempts have been made by researchers to delineate the role of immunity in the development of PBC granulomas. A recent comprehensive immunohistochemical analysis by You et al. [89] has shown that $\mathrm{CD} 11 \mathrm{c}$, the classical dendritic cell marker, is highly expressed in $\mathrm{PBC}$ granulomas. It also appears that CD11c-positive epitheliod granulomas are more prevalent in patients with PBC at early disease stages. Finally, the expression of CD11c in $\mathrm{PBC}$ granulomas is associated with higher IgM levels. These findings further support the notion that the influence of antigenic stimulation to professional antigen presenting cells largely influences the formation of granulomas in PBC. You et al. [89] suggested that $\mathrm{PBC}$ liver granulomas may result from the interaction between immature dendritic cells and $\operatorname{IgM}$, but this needs to be addressed at the experimental level. On the other hand, in human tuberculosis the formation of granulomas is considered either as an attempt to contain M. tuberculosis infection or an early effort by the pathogenic mycobacteria to assist the spreading of bacteria to uninfected macrophages that are recruited at the site of inflammation $[66,90]$. This perception raises the notion that granulomas in human tuberculosis may have a beneficial or catastrophic potential for the host depending on the timing of their formation $[91,92]$.

2.1. Evidence of Mycobacterial Infection in PBC. Tanaka et al. searched for evidence of infectious agents by PCR in livers 
TABLE 1: Summary of the major findings of studies investigating the role of Mycobacterium tuberculosis as a trigger of PBC. The findings are presented in relation to Koch's postulates. To date, no conclusive evidence has been presented which links Mycobacterium tuberculosis with PBC.

\begin{tabular}{|c|c|c|}
\hline Koch's postulates & Finding & Study \\
\hline $\begin{array}{l}\text { Infectious aetiology } \\
\text { Microorganism must be present in every } \\
\text { case of diseased individuals }\end{array}$ & $\begin{array}{l}\text { Positive staining for mycobacterial hsp65 in PBC cases has been } \\
\text { demonstrated, but hsp65 is conserved among all mycobacterial } \\
\text { species and it is unclear whether this staining is Mycobacterium } \\
\text { tuberculosis-specific and characteristic for PBC patients with } \\
\text { granulomata }\end{array}$ & {$[75]$} \\
\hline $\begin{array}{l}\text { Isolation } \\
\text { The microorganism must be isolated and } \\
\text { grown in pure cultures }\end{array}$ & No such data exist & \\
\hline $\begin{array}{l}\text { Disease causality } \\
\text { Pure cultures inoculated in healthy } \\
\text { animals must reproduce the disease }\end{array}$ & $\begin{array}{l}\text { No such data exist } \\
\text { Only one case report of a female that developed PBC following } \\
\text { tuberculosis infection has been published } \\
\text { There is no epidemiological or evidence linking tuberculosis with } \\
\text { PBC, including vaccination against tuberculosis }\end{array}$ & $\begin{array}{c}{[76]} \\
{[77-80]}\end{array}$ \\
\hline $\begin{array}{l}\text { Reproducibility } \\
\text { The microorganism must be recovered } \\
\text { from the diseased animal }\end{array}$ & No such data exist & \\
\hline $\begin{array}{l}\text { Other data indirectly relevant to Koch's } \\
\text { postulates }\end{array}$ & $\begin{array}{l}\text { PBC specific AMA was detected in } 43 \% \text { of tuberculosis patients, but } \\
\text { in low titres, and did not show the typical indirect immunofluorescent } \\
\text { patterns of antimitochondrial antibodies seen in PBC. These patients } \\
\text { do not have clinical features of PBC. }\end{array}$ & $\begin{array}{l}{[19,21,22,} \\
\quad 81]\end{array}$ \\
\hline
\end{tabular}

tissues from 29 patients with PBC. Mycobacterial DNA was not detected in any of these samples [93]. Other attempts to provide evidence of mycobacteria DNA in livers from PBC cases have provided inconclusive data. Broome et al. [75] demonstrated positive staining for mycobacterial hsp65 using a monoclonal antibodies in nine of the ten PBC cases [75]. O'Donohue and colleagues [94] studied liver biopsy specimen from eleven PBC cases. Five lymph nodes from patients with tuberculous lymphadenopathy were used as positive controls [94]. Three of the positive controls also had liver biopsies taken for concurrent tuberculous hepatitis. Four of the five positive controls had detectable mycobacterial DNA. Mycobacterial DNA was undetectable in the tissues obtained from patients with PBC [94].

\subsection{PBC-Specific Autoantibodies in Patients with Tuberculosis.} An early study by Klein et al. [81] reported the presence of PBC-specific AMA in 12/28 (43\%) of patients with tuberculosis. Immunoblotting demonstrated that these sera recognized PDC-E2 based on purified mitochondrial fraction derived from beef heart mitochondrial as an antigenic source. Only $2 \%$ of sera from individuals with other viral and bacterial infections showed reactivity with PDC-E2, and there was no reaction with sera from healthy controls [81]. As well, the titres of anti-PDC-E2 antibodies were low [81]. Additionally, none of the anti-PDC-E2 antibody positive sera (1/10 dilution) gave an immunofluorescent pattern typical of PBC by indirect immunofluorescence [19, 21, 22, 81]. This contrasts to what is normally seen in most patients with PBC, where titers of PDC-E2 targeting AMA give typical and strong immunofluorescent staining at dilutions as high as $1 / 100,000$. Amongst the 12 AMA positive cases with active tuberculosis, six had abnormal levels of $\gamma \mathrm{GT}$ and four had elevated levels of alkaline phosphatase, with only two of the twelve cases having increased IgM levels. None of the patients had increased bilirubin levels or evidence of impaired synthetic function. Liver biopsies were not performed and it is not known what has happen to these individuals over the years, and whether they have developed clinically overt liver disease. There was no information as to whether other causes of abnormal liver biochemistry such as drug-induced hepatotoxicity were excluded.

Patients with M. tuberculosis and AMA positivity show reactivity to $\mathrm{PDC}-\mathrm{E} 2$, which has not been observed in those infected with other forms of mycobacteria [81, 95]. Gilburd et al. [95] suggested sequence homology between $M$. leprae antigens and the 35,41 , and $54 \mathrm{kDa}$ subunits of OADC, implying that molecular mimicry and immunological crossreactivity between $M$. leprae and human mitochondrial autoantigens may be responsible for the induction of AMA in patients with leprosy.

\subsection{Mycobacteria and PBC: The Role of Molecular Mimicry.} It is unclear as to whether a negative result for $M$. tuberculosis also rules out the effects of a previous and nonactive infection. It has been suggested that mycobacteria and other infectious agents may be involved in the initiation of autoimmunity by microbial/self-immunological crossreactivity, where infection and clearance of the infection occur before the onset of clinical disease [96-100]. The role of molecular mimicry has been studied by several groups [96, 101-107] and immunological cross-reactivity has been documented [108-113] as a mechanism responsible for the induction of autoantigen-specific immune responses in 
microbial-triggered autoimmunity $[107,114-117]$ in susceptible individuals $[118,119]$. Impairment in the immunosuppressory functions of the host appears to be important for the induction and perpetuation of autoaggression induced via molecular mimicry or other mechanisms [120, 121]. Indeed, our group has studied molecular mimicry as a potential inducer of autoimmunity in several autoimmune liver and gastrointestinal diseases [97, 104, 110, 122, 123]. Most of the microbial/self-homologues are not targets of cross-reactive responses, and this underlines the importance of diseasespecific pairs targeted by antibodies $[99,100,111,113,124$, 125]. Of relevance to mycobacteria, Vilagut et al. [126] has reported that hsp65 $\mathrm{kDa} M$. gordonae and human OADC antigens, including human PDC-E2, are cross-reactive [127]. They also reported the presence of mycobacterial DNA in livers from patients with PBC. We have attempted to better define the extent of this cross-reactivity at the peptidyl level and through amino-acid comparison database searches, and we have identified an amino-acid homology between human PDC-E2, and mycobacterial hsp65 [124]. Thus, the hexameric motif [GDL(IL)AE)] is present in hsp6594-99 and the major PBC-specific mitochondrial autoepitopes, namely, the inner lipoyl PDC-E2 $216-221$ and the outer lipoyl domain human PDC-E2 $102-107$ [124]. The hsp65 mimic was not restricted to $M$. gordonae, but was conserved amongst mycobacteria (including $M$. tuberculosis). The SxGDL[IL]AE motif is virtually unique to mycobacterial hsp, and human PDC-E2 is the only known human sequence containing it [124]. We also obtained data to suggest that peptides spanning the homologous mycobacterial hsp65 and human PDC-E2 sequences are targets of cross-reactive responses when serum samples from patients with $\mathrm{PBC}$ are tested. These data support the notion that mycobacterial infection could initiate antimycobacterial responses against hsp65, which in turn could cross-react with human PDC-E2. The relevance of the biological significance of these data in the immunopathogenesis of microbial-induced $\mathrm{PBC}$ remains unaddressed.

If there is a link between tuberculosis and PBC, it would be expected that areas where $M$. tuberculosis is endemic would have high rates of $\mathrm{PBC}$, but this is not the case. For example, southern Africa is endemic for tuberculosis, but $\mathrm{PBC}$ is relatively rare in those regions $[128,129]$. The same could be said for India and China, which have relatively high rates of tuberculosis but low rates of PBC [130-132]. Also, epidemiological studies assessing susceptibility to $\mathrm{PBC}$ would be expected to identify tuberculosis as a risk factor.

\section{Risk Factors of PBC: Epidemiological Studies}

Several epidemiological studies have been conducted to investigate risk factors for the development of PBC [7780]. These studies have largely been based on questionnaires addressing geographical and lifestyle factors, as well as personal and familial medical and surgical histories. However, very few of these studies specifically mention infection with M. tuberculosis. A study by Parikh-Patel and colleagues
[79] administered a standardized US National Health and Nutrition Examination Study (NHANES) questionnaire to 241 PBC patients from the USA, in addition to 261 of their siblings and 141 friends as controls. Within the medical histories, it was found that 2 patients with $\mathrm{PBC}$ (1.2\%) reported having tuberculosis as adults, compared to none of the siblings, and one friend (0.8\%) [79]. A larger study conducted by Gershwin et al. [78] was also based on an NHANES questionnaire. The cohort in that study consisted of 1032 PBC patients from 23 tertiary care centres in the USA, 1041 controls selected from a random-digit-dialling protocol, which were sex, age, race, and geographically matched. All participants were administered the questionnaire by trained professionals, but it was not indicated as to whether specific questions were asked regarding tuberculosis [78]. However, it appears that questions were raised as to whether participants had been vaccinated for tuberculosis [78]. Despite this, it does not appear that any significant link was found in regards to tuberculosis and/or tuberculosis infection [78]. Prince et al. [80] conducted an epidemiological study involving $318 \mathrm{PBC}$ patients from an epidemiological study and 2258 from a PBC support group, in addition to 2438 controls. There was no indication as to whether specific questions were raised in regards to tuberculosis [80]. Corpechot and colleagues [77] administered a standardized questionnaire to $222 \mathrm{PBC}$ patients and 509 age, sex, and residentially matched controls [77]. Although no history of previous tuberculosis infection was indicated, it appears that questions regarding immunization for tuberculosis were asked, as $1 \%$ of PBC cases reported vaccination, although $1 \%$ of controls also reported vaccination [77]. Based on the epidemiological evidence, it does not appear that infection with $M$. tuberculosis is linked with PBC. However, it should also be noted that it is not clear as to whether history of tuberculosis infection and/or vaccination was investigated in some studies. In those which do, there was no significantly higher incidence of $\mathrm{PBC}$ infection among patients compared to controls, and there was no significantly higher incidence of vaccination against tuberculosis in PBC patients. An association between vaccination and the development of $\mathrm{PBC}$ has been reported for other microbial agents, and in particular Lactobacilli, and the mechanism of molecular mimicry has been proposed to account for the induction of PBC-specific autoreactivity $[97,98]$.

\section{Case Studies of PBC and Tuberculosis}

A PubMed search for case reports in of patients with tuberculosis who developed PBC (search terms: "primary biliary cirrhosis, tuberculosis" and "primary biliary cirrhosis, mycobacterium tuberculosis") revealed one case [76]. That case reports of a 70-year-old Tunisian patient who developed antimitochondrial antibodies and anti-ADN during urogenital tuberculosis. There were clinical and biological signs of primary biliary cirrhosis and systemic lupus erythematosus in that patient [76]. If there was a link between tuberculosis and $\mathrm{PBC}$, it would be expected that several case reports 
of patients developing PBC, or AMA positivity following infection with $M$. tuberculosis.

\section{Genome-Wide Association Studies in Tuberculosis and in PBC}

Genome-wide association studies (GWAS) have recently shed light on the genetic background of $\mathrm{PBC}$ and pulmonary tuberculosis. Genes implicated in PBC lay within both HLA and non-HLA regions (Table 2) [133-140]. GWAS have led to the identification of several genes that infer susceptibility to developing active pulmonary tuberculosis 1 [141, 142] including JAG1, DYNLRB2, EBF1, TMEFF2, CCL17, HAUS6, PENK, and TXNDC4. If tuberculosis is a risk factor for the development of $\mathrm{PBC}$, it would be reasonable to infer that both conditions would share several susceptibility genes [143]. However, no significant overlapping of genes conferring susceptibility to both $\mathrm{PBC}$ and tuberculosis has been identified (Table 2). Also depending on the geographical region, environmental exposures related to tuberculosis may differ, as well as strong associations between different evolutionary lineages of Mycobacterium tuberculosis with specific geographical regions have been noted [144]. Such differences have been extensively studied in tuberculosis patients, such as those originating from Indonesian patients living in Jakarta and Bandung compared to other regions/islands. The extent by which genetic coevolution and phylogenetic difference involving M. tuberculosis participate in the dysregulation of host's immune response is worthy for further investigation.

\section{Tuberculosis in Other Autoimmune Diseases}

$\mathrm{PBC}$ is not the only autoimmune disease in which $M$. tuberculosis has been implicated. M. Tuberculosis has also been noted to be involved in a more common autoimmune disease, SLE, and specifically to disease flares. An early study by Shoenfeld and colleagues [73] examined whether murine monoclonal anti-TB antibodies reacted with ssDNA, dsDNA, and other polynucleotides and found that monoclonal anti-DNA autoantibodies from humans and mouse SLE models bound to three glycolipids shared among mycobacteria. Incubation of the antibodies with ssDNA and/or polynucleotides or glycolipid antigens was found to inhibit binding [73]. This suggests a possible explanation for the production of autoantibodies in mycobacterial infections. Sela and colleagues [145] examined the sera from 57 patients with untreated TB for the anti-DNA idiotype 16/6 and found that $60 \%$ had increased levels of the idiotype compared to $4 \%$ of controls. As well, there were also increased levels of autoantibodies such as those to ssDNA, dsDNA, polynucleotides, and cardiolipin [145]. A study by Amital-Teplizki et al. [67] examined the binding of human lupus anti-DNA antibodies and murine antimycobacterial antibodies to human cortical brain tissue. That group found that antimycobacterial and anti-DNA antibodies competed on the binding site of a common neuronal membrane
TABLE 2: Major susceptibility genes associated with tuberculosis $[141,142]$ and primary biliary cirrhosis [133-140], as reported through genome wide association studies. Note that no positive associations are significantly shared between primary biliary cirrhosis (PBC) and pulmonary tuberculosis (TB).

\begin{tabular}{lcc}
\hline Gene & PBC & TB \\
\hline HLA & + & - \\
DR8 & + & - \\
DQB1 & + & - \\
DRB1 & + & - \\
DQA1 & + & - \\
DQA2 & & \\
Non-HLA & + & - \\
STAT4 & + & - \\
SPIB & + & - \\
IRF5 & + & - \\
IL12A & + & - \\
IL12RB & + & - \\
MMEL1 & + & - \\
CXCR5 & + & + \\
NFKB1 & - & + \\
JAG1 & - & + \\
DYNLRB2 & - & + \\
EBF1 & - & + \\
TMEFF2 & - & + \\
CCL17 & - & + \\
HAUS6 & - & + \\
PENK & - & + \\
TXNDC4 & - & + \\
\hline
\end{tabular}

epitope, indicating the presence of a shared antigen between mycobacteria and DNA [67].

In addition to the above molecular findings, a link between SLE flares and TB have also been noted. Ribeiro and colleagues [74] describe the development of TB infection in parallel with lupus flares in four females. The site of mycobacterial infection/detection in the women included a positive urine culture for mycobacterial spp., a nodular pulmonary lesion with subsequent positive tuberculin cutaneous test, a positive acid-fact bacilli test on bronchoalveolar lavage, and one case of disseminated TB [74]. In all four women, SLE flares did not improve on administration of antirheumatic treatment and immunosuppression but did improve after the administration of antituberculosis treatment [74]. Those authors suggest that M. tuberculosis stimulated the production of autoantibodies with shared affinity for mycobacterial and human antigens, which in turn may have led to the SLE flare [74]. These cases indicate a possible link between SLE and TB, but it should also be noted that the immunosuppressive treatment given to SLE patients may in fact reactivate a latent TB infection.

\section{Conclusion}

The study of the role of Mycobacterium tuberculosis in PBC has been limited due to the scarcity of studies and data. 
Multiple epidemiological studies have failed to indicate tuberculosis as a risk factor for the development of PBC, and there is an absence of case reports of tuberculosis infection followed by PBC in the literature. It would be expected that if $M$. tuberculosis was linked with $\mathrm{PBC}$, there would be a higher incidence of $\mathrm{PBC}$ in areas which are endemic for tuberculosis. However, this does not appear to be the case [146]. It would also be expected that if $M$. tuberculosis was linked to $\mathrm{PBC}$, several genes which predispose to one disease, would overlap with the other. However, with the advent of GWAS, this link has also been ruled out, with no genes being shared between the two conditions. Although more studies are needed to investigate a potential link, the existent data are highly suggestive of a lack of a causative link between tuberculosis and PBC. However, the observation of granulomatous lesion in $\mathrm{PBC}$ liver biopsies warrants further investigation, as the significance of these lesions in the liver of PBC patients is unknown.

\section{Author's Contribution}

E. I. Rigopoulou and D. P. Bogdanos conceived the idea for writing a review on this topic; D. S. Smyk, D. P. Bogdanos, and E. I. Rigopoulou wrote the first and subsequent drafts of the paper. All authors contributed to the editing of the first and subsequent versions of the paper. All authors have read and approved the final paper.

\section{References}

[1] S. Hohenester, R. P. J. Oude-Elferink, and U. Beuers, "Primary biliary cirrhosis," Seminars in Immunopathology, vol. 31, no. 3, pp. 283-307, 2009.

[2] M. M. Kaplan and M. E. Gershwin, "Primary biliary cirrhosis," The New England Journal of Medicine, vol. 353, no. 12, pp. 1261-1273, 2005.

[3] T. Kumagi and E. J. Heathcote, "Primary biliary cirrhosis," Orphanet Journal of Rare Diseases, vol. 3, no. 1, article no. 1, 2008.

[4] K. D. Lindor, M. E. Gershwin, R. Poupon, M. Kaplan, N. V. Bergasa, and E. J. Heathcote, "Primary biliary cirrhosis," Hepatology, vol. 50, no. 1, pp. 291-308, 2009.

[5] J. Neuberger, "Primary biliary cirrhosis," The Lancet, vol. 350, no. 9081, pp. 875-879, 1997.

[6] E. Jenny Heathcote, "Management of primary biliary cirrhosis. The American Association for the Study of Liver Diseases practice guidelines," Hepatology, vol. 31, no. 4, pp. 10051013, 2000.

[7] R. Poupon, "Primary biliary cirrhosis: a 2010 update," Journal of Hepatology, vol. 52, no. 5, pp. 745-758, 2010.

[8] D. S. Smyk, E. I. Rigopoulou, A. Pares et al., "Sex differences associated with primary biliary cirrhosis," Clinical and developmental immunology, vol. 2012, Article ID 610504, 2012.

[9] P. Invernizzi, M. G. Alessio, D. S. Smyk et al., "Autoimmune hepatitis type 2 associated with an unexpected and transient presence of primary biliary cirrhosis-specific antimitochondrial antibodies: a case study and review of the literature," BMC Gastroenterology, vol. 12, p. 92, 2012.

[10] C. A. Aoki, C. M. Roifman, Z. X. Lian et al., "IL-2 receptor alpha deficiency and features of primary biliary cirrhosis," Journal of Autoimmunity, vol. 27, no. 1, pp. 50-53, 2006.
[11] Y. Dahlan, L. Smith, D. Simmonds et al., "Pediatric-onset primary biliary cirrhosis," Gastroenterology, vol. 125, no. 5, pp. 1476-1479, 2003.

[12] D. Smyk, E. Cholongitas, S. Kriese, E. I. Rigopoulou, and D. P. Bogdanos, "Primary biliary cirrhosis: family stories," Autoimmune Diseases, vol. 2011, Article ID 189585, 2011.

[13] D. Smyk, E. I. Rigopoulou, A. Pares, M. Mytilinaiou, P. Invernizzi, and D. Bogdanos, "Familial primary biliary cirrhosis: like mother, like daughter?" Acta Gastro-Enterologica Belgica, vol. 75, pp. 203-209, 2012.

[14] K. Boonstra, U. Beuers, and C. Y. Ponsioen, "Epidemiology of primary sclerosing cholangitis and primary biliary cirrhosis: a systematic review," Journal of Hepatology, vol. 56, pp. 1181$1188,2012$.

[15] O. E. W. James, R. Bhopal, D. Howel, J. Gray, A. D. Burt, and J. V. Metcalf, "Primary biliary cirrhosis once rare, now common in the United Kingdom?” Hepatology, vol. 30, no. 2, pp. 390-394, 1999.

[16] W. R. Kim, K. D. Lindor, G. R. Locke et al., "Epidemiology and natural history of primary biliary cirrhosis in a U.S. community," Gastroenterology, vol. 119, no. 6, pp. 16311636, 2000.

[17] S. Sood, P. J. Gow, J. M. Christie, and P. W. Angus, "Epidemiology of primary biliary cirrhosis in Victoria, Australia: high prevalence in migrant populations," Gastroenterology, vol. 127, no. 2, pp. 470-475, 2004.

[18] C. Selmi, P. Invernizzi, E. B. Keefe et al., "Epidemiology and pathogenesis of primary biliary cirrhosis," Journal of Clinical Gastroenterology, vol. 38, no. 3, pp. 264-271, 2004.

[19] D. P. Bogdanos, G. Mieli-Vergani, and D. Vergani, "Autoantibodies and their antigens in autoimmune hepatitis," Seminars in Liver Disease, vol. 29, no. 3, pp. 241-253, 2009.

[20] D. P. Bogdanos, H. Baum, and D. Vergani, "Antimitochondrial and other autoantibodies," Clinics in Liver Disease, vol. 7, no. 4, pp. 759-777, 2003.

[21] D. P. Bogdanos, P. Invernizzi, I. R. Mackay, and D. Vergani, "Autoimmune liver serology: current diagnostic and clinical challenges," World Journal of Gastroenterology, vol. 14, no. 21, pp. 3374-3387, 2008.

[22] D. P. Bogdanos and L. Komorowski, "Disease-specific autoantibodies in primary biliary cirrhosis," Clinica Chimica Acta, vol. 412, no. 7-8, pp. 502-512, 2011.

[23] D. P. Bogdanos, C. Liaskos, E. I. Rigopoulou, and G. N. Dalekos, "Anti-mitochondrial antibodies in patients with systemic lupus erythematosus: revealing the unforeseen," Clinica Chimica Acta, vol. 373, no. 1-2, pp. 183-184, 2006.

[24] P. S. C. Leung, R. L. Coppel, A. Ansari, S. Munoz, and M. E. Gershwin, "Antimitochondrial antibodies in primary biliary cirrhosis," Seminars in Liver Disease, vol. 17, no. 1, pp. 61-69, 1997.

[25] D. P. Bogdanos, A. Pares, J. Rodés et al., "Primary biliary cirrhosis specific antinuclear antibodies in patients from Spain," American Journal of Gastroenterology, vol. 99, no. 4, pp. 763-765, 2004.

[26] D. P. Bogdanos, D. Vergani, P. Muratori, L. Muratori, and F. B. Bianchi, "Specificity of anti-sp100 antibody for primary biliary cirrhosis," Scandinavian Journal of Gastroenterology, vol. 39, no. 4, pp. 405-407, 2004.

[27] P. Invernizzi, M. Podda, P. M. Battezzati et al., "Autoantibodies against nuclear pore complexes are associated with more active and severe liver disease in primary biliary cirrhosis," Journal of Hepatology, vol. 34, no. 3, pp. 366-372, 2001.

[28] L. Muratori, A. Granito, P. Muratori, G. Pappas, and F. B. Bianchi, "Antimitochondrial antibodies and other antibodies 
in primary biliary cirrhosis: diagnostic and prognostic value," Clinics in Liver Disease, vol. 12, no. 2, pp. 261-276, 2008.

[29] C. Rigamonti, D. P. Bogdanos, M. G. Mytilinaiou, D. S. Smyk, E. I. Rigopoulou, and A. K. Burroughs, "Primary biliary cirrhosis associated with systemic sclerosis: diagnostic and clinical challenges," International Journal of Rheumatology, vol. 2011, Article ID 976427, 12 pages, 2011.

[30] D. P. Bogdanos, C. Rigamonti, D. Smyk, M. G. Mytilinaiou, E. I. Rigopoulou, and A. K. Burroughs, "Emerging issues in the immunopathogenesis, diagnosis and clinical management of primary biliary cirrhosis associated with systemic sclerosis," in Systemic Sclerosis-An Update on the Aberrant Immune System and Clinical Features, T. Radstake, Ed., pp. 151-166, Intech, Rijeka, Croatia, 2012.

[31] T. Kumagi and M. Onji, "Presentation and diagnosis of primary biliary sirrhosis in the 21st century," Clinics in Liver Disease, vol. 12, no. 2, pp. 243-259, 2008.

[32] C. Duarte-Rey, D. P. Bogdanos, P. S. Leung, J. M. Anaya, and M. E. Gershwin, "IgM predominance in autoimmune disease: genetics and gender," Autoimmun Rev, vol. 11, pp. A404-A412, 2012.

[33] E. I. Rigopoulou, E. T. Davies, D. P. Bogdanos et al., "Antimitochondrial antibodies of immunoglobulin G3 subclass are associated with a more severe disease course in primary biliary cirrhosis," Liver International, vol. 27, no. 9, pp. 12261231, 2007.

[34] E. I. Rigopoulou, D. P. Bogdanos, C. Liaskos et al., "Antimitochondrial antibody immunofluorescent titres correlate with the number and intensity of immunoblot-detected mitochondrial bands in patients with primary biliary cirrhosis," Clinica Chimica Acta, vol. 380, no. 1-2, pp. 118-121, 2007.

[35] C. Dähnrich, A. Pares, L. Caballeria et al., "New ELISA for detecting primary biliary cirrhosis-specific antimitochondrial antibodies," Clinical Chemistry, vol. 55, no. 5, pp. 978985, 2009.

[36] P. Invernizzi, A. Crosignani, P. M. Battezzati et al., "Comparison of the clinical features and clinical course of antimitochondrial antibody-positive and -negative primary biliary cirrhosis," Hepatology, vol. 25, no. 5, pp. 1090-1095, 1997.

[37] N. Bizzaro, G. Covini, F. Rosina et al., "Overcoming a "probable" diagnosis in antimitochondrial antibody negative primary biliary cirrhosis: study of 100 sera and review of the literature," Clinical Reviews in Allergy and Immunology, vol. 42, no. 3, pp. 288-297, 2012.

[38] W. Bernal, F. Meda, Y. Ma, D. P. Bogdanos, and D. Vergani, "Disease-specific autoantibodies in patients with acute liver failure: the King's College London experience," Hepatology, vol. 47, no. 3, pp. 1096-1097, 2008.

[39] P. S. C. Leung, L. Rossaro, P. A. Davis et al., "Antimitochondrial antibodies in acute liver failure: implications for primary biliary cirrhosis," Hepatology, vol. 46, no. 5, pp. 1436-1442, 2007.

[40] C. Liaskos, D. P. Bogdanos, E. I. Rigopoulou, and G. N. Dalekos, "Development of antimitochondrial antibodies in patients with autoimmune hepatitis: art of facts or an artifact?" Journal of Gastroenterology and Hepatology, vol. 22, no. 3, pp. 454-455, 2007.

[41] J. V. Metcalf, H. C. Mitchison, J. M. Palmer, D. E. Jones, M. F. Bassendine, and O. F. W. James, "Natural history of early primary biliary cirrhosis," The Lancet, vol. 348, no. 9039, pp. 1399-1402, 1996.
[42] C. Duarte-Rey, D. Bogdanos, C. Y. Yang et al., "Primary biliary cirrhosis and the nuclear pore complex," Autoimmunity Reviews, vol. 11, no. 12, pp. 898-902, 2012.

[43] S. Itoh, T. Ichida, T. Yoshida et al., "Autoantibodies against a $210 \mathrm{kDa}$ glycoprotein of the nuclear pore complex as a prognostic marker in patients with primary biliary cirrhosis," Journal of Gastroenterology and Hepatology, vol. 13, no. 3, pp. 257-265, 1998.

[44] J. Wesierska-Gadek, H. Hohenauer, E. Hitchman, and E. Penner, "Autoantibodies from patients with primary biliary cirrhosis preferentially react with the amino-terminal domain of nuclear pore complex glycoprotein gp210," Journal of Experimental Medicine, vol. 182, no. 4, pp. 1159-1162, 1995.

[45] E. I. Rigopoulou, E. T. Davies, A. Pares et al., "Prevalence and clinical significance of isotype specific antinuclear antibodies in primary biliary cirrhosis," Gut, vol. 54, no. 4, pp. 528-532, 2005.

[46] D. P. Bogdanos, C. Liaskos, A. Pares et al., "Anti-gp210 antibody mirrors disease severity in primary biliary cirrhosis," Hepatology, vol. 45, no. 6, p. 1583, 2007.

[47] M. G. Mytilinaiou, W. Meyer, T. Scheper et al., "Diagnostic and clinical utility of antibodies against the nuclear body promyelocytic leukaemia and Sp100 antigens in patients with primary biliary cirrhosis," Clinica Chimica Acta, vol. 413, pp. 1211-1216, 2012.

[48] C. Liaskos, G. L. Norman, A. Moulas et al., "Prevalence of gastric parietal cell antibodies and intrinsic factor antibodies in primary biliary cirrhosis," Clinica Chimica Acta, vol. 411, no. 5-6, pp. 411-415, 2010.

[49] S. Gabeta, G. L. Norman, N. Gatselis et al., "IgA anti-b2GPI antibodies in patients with autoimmune liver diseases," Journal of Clinical Immunology, vol. 28, no. 5, pp. 501-511, 2008.

[50] P. Muratori, L. Muratori, M. Guidi et al., "Anti-Saccharomyces cerevisiae antibodies (ASCA) and autoimmune liver diseases," Clinical and Experimental Immunology, vol. 132, no. 3, pp. 473-476, 2003.

[51] E. I. Rigopoulou, D. Roggenbuck, D. S. Smyk et al., "Asialoglycoprotein receptor (ASGPR) as target autoantigen in liver autoimmunity: lost and found," Autoimmunity Reviews. In press.

[52] D. P. Bogdanos, D. S. Smyk, E. I. Rigopoulou et al., "Twin studies in autoimmune disease: genetics, gender and environment," Journal of Autoimmunity, vol. 38, pp. J156J169, 2012.

[53] C. Selmi, P. S. Leung, D. H. Sherr et al., "Mechanisms of environmental influence on human autoimmunity: a national institute of environmental health sciences expert panel workshop," Journal of Autoimmunity. In press.

[54] F. W. Miller, K. M. Pollard, C. G. Parks et al., "Criteria for environmentally associated Autoimmune Dis," Journal of Autoimmunity. In press.

[55] D. S. Smyk, E. I. Rigopoulou, L. Muratori, A. K. Burroughs, and D. P. Bogdanos, "Smoking as a risk factor for autoimmune liver disease: what we can learn from primary biliary cirrhosis," Annals of Hepatology, vol. 11, pp. 7-14, 2012.

[56] D. S. Smyk, M. G. Mytilinaiou, P. Milkiewicz, E. I. Rigopoulou, P. Invernizzi, and D. P. Bogdanos, "Towards systemic sclerosis and away from primary biliary cirrhosis: the case of PTPN22," Autoimmunity Highlights, vol. 3, pp. 19, 2012.

[57] D. S. Smyk, E. I. Rigopoulou, N. Bizarro, and D. P. Bogdanos, "Hair dyes as a risk for autoimmunity: from systemic lupus 
erythematosus to primary biliary cirrhosis," Autoimmunity Highlights. In press.

[58] D. Smyk, M. G. Mytilinaiou, E. I. Rigopoulou, and D. P. Bogdanos, "PBC triggers in water reservoirs, coal mining areas and waste disposal sites: from Newcastle to New York," Disease Markers, vol. 29, no. 6, pp. 337-344, 2010.

[59] D. S. Smyk, E. I. Rigopoulou, A. Lleo et al., "Immunopathogenesis of primary biliary cirrhosis: an old wives' tale," Immunity \& Ageing, vol. 8, p. 12, 2011.

[60] E. I. Rigopoulou, D. S. Smyk, C. E. Matthews et al., "Epsteinbarr virus as a trigger of autoimmune liver diseases," Advances in Virology, vol. 2012, Article ID 987471, 12 pages, 2012.

[61] D. S. Smyk, D. P. Bogdanos, S. Kriese, C. Billinis, A. K. Burroughs, and E. I. Rigopoulou, "Urinary tract infection as a risk factor for autoimmune liver disease: from bench to bedside," Clinics and Research in Hepatology and Gastroenterology, vol. 36, pp. 110-121, 2012.

[62] D. Smyk, E. I. Rigopoulou, H. Baum, A. K. Burroughs, D. Vergani, and D. P. Bogdanos, "Autoimmunity and environment: am i at risk?" Clinical Reviews in Allergy and Immunology, vol. 42, pp. 199-212, 2012.

[63] F. K. Varyani, J. West, and T. R. Card, "An increased risk of urinary tract infection precedes development of primary biliary cirrhosis," BMC Gastroenterology, vol. 11, p. 95, 2011.

[64] D. Smyk, E. I. Rigopoulou, Z. Yoh, A. Koutsoumpas, H. Baum, and D. P. Bogdanos, "Infectious triggers of primary biliary cirrhosis: do we know enough?" Current Trends in Immunology, vol. 11, pp. 35-49, 2010.

[65] G. Maartens and R. J. Wilkinson, "Tuberculosis," The Lancet, vol. 370, no. 9604, pp. 2030-2043, 2007.

[66] B. M. Saunders and W. J. Britton, "Life and death in the granuloma: immunopathology of tuberculosis," Immunology and Cell Biology, vol. 85, no. 2, pp. 103-111, 2007.

[67] H. Amital-Teplizki, I. Avinoach, A. R. M. Coates, O. Kooperman, M. Blank, and Y. Shoenfeld, "Binding of monoclonal anti-DNA and anti-TB glycolipids to brain tissue," Autoimmunity, vol. 4, no. 4, pp. 277-287, 1989.

[68] R. A. Asherson, K. Gunter, D. Daya, and Y. Shoenfeld, "Multiple autoimmune diseases in a young woman: tuberculosis and splenectomy as possible triggering factors? Another example of the "mosaic" of autoimmunity," Journal of Rheumatology, vol. 35, no. 6, pp. 1224-1227, 2008.

[69] M. Cutolo, C. Pizzorni, and A. Sulli, "Vitamin D endocrine system involvement in autoimmune rheumatic diseases," Autoimmunity Reviews, vol. 11, pp. 84-87, 2011.

[70] A. Dubaniewicz, "Mycobacterium tuberculosis heat shock proteins and autoimmunity in sarcoidosis," Autoimmunity Reviews, vol. 9, no. 6, pp. 419-424, 2010.

[71] Y. Shapira, N. Agmon-Levin, and Y. Shoenfeld, "Mycobacterium tuberculosis, autoimmunity, and vitamin D," Clinical Reviews in Allergy and Immunology, vol. 38, no. 2-3, pp. 169$177,2010$.

[72] M. A. B. Thomas, G. Frampton, D. A. Isenberg et al., "A common anti-DNA antibody idiotype and anti-phospholipid antibodies in sera from patients with schistosomiasis and filariasis with and without nephritis," Journal of Autoimmunity, vol. 2, no. 6, pp. 803-811, 1989.

[73] Y. Shoenfeld, Y. Vilner, and A. R. M. Coates, "Monoclonal anti-tuberculosis antibodies react with DNA, and monoclonal anti-DNA autoantibodies react with Mycobacterium tuberculosis," Clinical and Experimental Immunology, vol. 66, no. 2, pp. 255-261, 1986.
[74] F. M. Ribeiro, M. Szyper-Kravitz, E. M. Klumb et al., "Can lupus flares be associated with tuberculosis infection?" Clinical Reviews in Allergy and Immunology, vol. 38, no. 2-3, pp. 163-168, 2010.

[75] U. Broome, A. Scheynius, and R. Hultcrantz, "Induced expression of heat-shock protein on biliary epithelium in patients with primary sclerosing cholangitis and primary biliary cirrhosis," Hepatology, vol. 18, no. 2, pp. 298-303, 1993.

[76] M. Kallel Sellami, M. Zitouni, F. Zouiten, L. Laadhar, T. Ben Chaabane, and S. Makni, "Tuberculosis, primary biliary cirrhosis and autoimmunity," Bulletin de la Societe de Pathologie Exotique, vol. 94, no. 4, pp. 330-331, 2001.

[77] C. Corpechot, Y. Chrétien, O. Chazouillères, and R. Poupon, "Demographic, lifestyle, medical and familial factors associated with primary biliary cirrhosis," Journal of Hepatology, vol. 53, no. 1, pp. 162-169, 2010.

[78] M. E. Gershwin, C. Selmi, H. J. Worman et al., "Risk factors and comorbidities in primary biliary cirrhosis: a controlled interview-based study of 1032 patients," Hepatology, vol. 42, no. 5, pp. 1194-1202, 2005.

[79] A. Parikh-Patel, E. B. Gold, H. Worman, K. E. Krivy, and M. E. Gershwin, "Risk factors for primary biliary cirrhosis in a cohort of patients from the United States," Hepatology, vol. 33 , no. 1, pp. 16-21, 2001.

[80] M. I. Prince, S. J. Ducker, and O. F. W. James, "Case-control studies of risk factors for primary biliary cirrhosis in two United Kingdom populations," Gut, vol. 59, no. 4, pp. 508512, 2010.

[81] R. Klein, M. Wiebel, S. Engelhart, and P. A. Berg, "Sera from patients with tuberculosis recognize the M2a-epitope (E2-subunit of pyruvate dehydrogenase) specific for primary biliary cirrhosis," Clinical and Experimental Immunology, vol. 92, no. 2, pp. 308-316, 1993.

[82] D. R. Gaya, D. Thorburn, K. A. Oien, A. J. Morris, and A. J. Stanley, "Hepatic granulomas: a 10 year single centre experience," Journal of Clinical Pathology, vol. 56, no. 11, pp. 850-853, 2003.

[83] W. G. McCluggage and J. M. Sloan, "Hepatic granulomas in Northern Ireland: a thirteen year review," Histopathology, vol. 25, no. 3, pp. 219-228, 1994.

[84] I. K. Onal, O. Ersoy, M. Aydinli et al., "Hepatic granuloma in Turkish adults: a report of 13 cases," European Journal of Internal Medicine, vol. 19, no. 7, pp. 527-530, 2008.

[85] J. S. Sartin and R. C. Walker, "Granulomatous hepatitis: a retrospective review of 88 cases at the Mayo Clinic," Mayo Clinic Proceedings, vol. 66, no. 9, pp. 914-918, 1991.

[86] M. B. Satti, H. Al-Freihi, E. M. Ibrahim et al., "Hepatic granuloma in Saudi Arabia: a clinicopathological study of 59 cases," American Journal of Gastroenterology, vol. 85, no. 6, pp. 669-674, 1990.

[87] U. Drebber, H. U. Kasper, J. Ratering et al., "Hepatic granulomas: histological and molecular pathological approach to differential diagnosis-a study of 442 cases," Liver International, vol. 28, no. 6, pp. 828-834, 2008.

[88] S. P. Dourakis, R. Saramadou, A. Alexopoulou et al., "Hepatic granulomas: a 6-year experience in a single center in Greece," European Journal of Gastroenterology and Hepatology, vol. 19, no. 2, pp. 101-104, 2007.

[89] Z. You, Q. Wang, Z. Bian et al., "The immunopathology of liver granulomas in primary biliary cirrhosis," Journal of Autoimmunity, vol. 39, no. 3, pp. 216-221, 2012. 
[90] J. L. Flynn, J. Chan, and P. L. Lin, "Macrophages and control of granulomatous inflammation in tuberculosis," Mucosal Immunology, vol. 4, no. 3, pp. 271-278, 2011.

[91] J. M. Davis and L. Ramakrishnan, "The role of the granuloma in expansion and dissemination of early tuberculous infection," Cell, vol. 136, no. 1, pp. 37-49, 2009.

[92] L. Ramakrishnan, "Revisiting the role of the granuloma in tuberculosis," Nature Reviews Immunology, vol. 12, pp. 352366, 2012.

[93] A. Tanaka, T. P. Prindiville, R. Gish et al., "Are infectious agents involved in primary biliary cirrhosis? A PCR approach," Journal of Hepatology, vol. 31, no. 4, pp. 664-671, 1999.

[94] J. O’Donohue, H. Fidler, M. Garcia-Barcelo, K. Nouri-Aria, R. Williams, and J. McFadden, "Mycobacterial DNA not detected in liver sections from patients with primary billiary cirrhosis," Journal of Hepatology, vol. 28, no. 3, pp. 433-438, 1998.

[95] B. Gilburd, L. Ziporen, D. Zharhary et al., "Antimitochondrial (pyruvate dehydrogenase) antibodies in leprosy," Journal of Clinical Immunology, vol. 14, no. 1, pp. 14-19, 1994.

[96] J. Van de Water, H. Ishibashi, R. L. Coppel, and M. E. Gershwin, "Molecular mimicry and primary biliary cirrhosis: premises not promises," Hepatology, vol. 33, no. 4, pp. 771775, 2001.

[97] D. Bogdanos, T. Pusl, C. Rust, D. Vergani, and U. Beuers, "Primary biliary cirrhosis following lactobacillus vaccination for recurrent vaginitis," Journal of Hepatology, vol. 49, no. 3, pp. 466-473, 2008.

[98] D. P. Bogdanos, H. Baum, M. Okamoto et al., "Primary biliary cirrhosis is characterized by IgG3 antibodies crossreactive with the major mitochondrial autoepitope and its lactobacillus mimic," Hepatology, vol. 42, no. 2, pp. 458-465, 2005.

[99] D. P. Bogdanos, H. Baum, U. C. Sharma et al., "Antibodies against homologous microbial caseinolytic proteases $\mathrm{P}$ characterise primary biliary cirrhosis," Journal of Hepatology, vol. 36, no. 1, pp. 14-21, 2002.

[100] D. P. Bogdanos and D. Vergani, "Bacteria and primary biliary cirrhosis," Clinical Reviews in Allergy and Immunology, vol. 36, no. 1, pp. 30-39, 2009.

[101] D. P. Bogdanos, H. Baum, P. Butler et al., "Association between the primary biliary cirrhosis specific anti-sp100 antibodies and recurrent urinary tract infection," Digestive and Liver Disease, vol. 35, no. 11, pp. 801-805, 2003.

[102] D. P. Bogdanos, H. Baum, D. Vergani, and A. K. Burroughs, "The role of $E$. coli infection in the pathogenesis of primary biliary cirrhosis," Disease Markers, vol. 29, no. 6, pp. 301-311, 2010.

[103] A. Koutsoumpas, D. Polymeros, Z. Tsiamoulos et al., "Peculiar antibody reactivity to human connexin 37 and its microbial mimics in patients with Crohn's disease," Journal of Crohn's and Colitis, vol. 5, no. 2, pp. 101-109, 2011.

[104] G. V. Gregorio, K. Choudhuri, Y. Ma et al., "Mimicry between the hepatitis $\mathrm{C}$ virus polyprotein and antigenic targets of nuclear and smooth muscle antibodies in chronic hepatitis $\mathrm{C}$ virus infection," Clinical and Experimental Immunology, vol. 133, no. 3, pp. 404-413, 2003.

[105] G. H. Haydon and J. Neuberger, "PBC: an infectious disease?" Gut, vol. 47, no. 4, pp. 586-588, 2000.

[106] H. Kita, S. Matsumura, X. S. He et al., "Analysis of TCR antagonism and molecular mimicry of an HLA-A $* 0201$-restricted
CTL epitope in primary biliary cirrhosis," Hepatology, vol. 36, no. 4 I, pp. 918-926, 2002.

[107] Y. Ma, D. P. Bogdanos, M. J. Hussain et al., "Polyclonal T-cell responses to cytochrome P450IID6 are associated with disease activity in autoimmune hepatitis type 2," Gastroenterology, vol. 130, no. 3, pp. 868-882, 2006.

[108] D. Polymeros, D. P. Bogdanos, R. Day, D. Arioli, D. Vergani, and A. Forbes, "Does cross-reactivity between mycobacterium avium paratuberculosis and human intestinal antigens characterize Crohn's disease?" Gastroenterology, vol. 131, no. 1, pp. 85-96, 2006.

[109] H. Baum, D. P. Bogdanos, and D. Vergani, "Antibodies to Clp protease in primary biliary cirrhosis: possible role of a mimicking T-cell epitope," Journal of Hepatology, vol. 34, no. 5, pp. 785-787, 2001.

[110] N. Kerkar, K. Choudhuri, Y. Ma et al., "Cytochrome P4502D6193-212: a new immunodominant epitope and target of virus/self cross-reactivity in liver kidney microsomal autoantibody type 1-positive liver disease," Journal of Immunology, vol. 170, no. 3, pp. 1481-1489, 2003.

[111] A. Koutsoumpas, M. Mytilinaiou, D. Polymeros, G. N. Dalekos, and D. P. Bogdanos, "Anti-Helicobacter pylori antibody responses specific for VacA do not trigger primary biliary cirrhosis-specific antimitochondrial antibodies," European Journal of Gastroenterology and Hepatology, vol. 21, no. 10, p. 1220, 2009.

[112] M. S. Longhi, M. J. Hussain, D. P. Bogdanos et al., "Cytochrome P450IID6-specific CD8 T cell immune responses mirror disease activity in autoimmune hepatitis type 2," Hepatology, vol. 46, no. 2, pp. 472-484, 2007.

[113] D. P. Bogdanos, H. Baum, F. Gunsar et al., "Extensive homology between the major immunodominant mitochondrial antigen in primary biliary cirrhosis and Helicobacter pylori does not lead to immunological cross-reactivity," Scandinavian Journal of Gastroenterology, vol. 39, no. 10, pp. 981-987, 2004.

[114] S. Hannam, D. P. Bogdanos, E. T. Davies et al., "Neonatal liver disease associated with placental transfer of antimitochondrial antibodies," Autoimmunity, vol. 35, no. 8, pp. 545-550, 2002.

[115] Y. Ma, M. Meregalli, S. Hodges et al., "Alcohol dehydrogenase: an autoantibody target in patients with alcoholic liver disease," International Journal of Immunopathology and Pharmacology, vol. 18, no. 1, pp. 173-182, 2005.

[116] Y. Ma, M. Okamoto, M. G. Thomas et al., "Antibodies to conformational epitopes of soluble liver antigen define a severe form of autoimmune liver disease," Hepatology, vol. 35, no. 3, pp. 658-664, 2002.

[117] Y. Ma, M. G. Thomas, M. Okamoto et al., "Key residues of a major cytochrome P4502D6 epitope are located on the surface of the molecule," Journal of Immunology, vol. 169, no. 1, pp. 277-285, 2002.

[118] L. Muratori, D. P. Bogdanos, P. Muratori et al., "Susceptibility to thyroid disorders in hepatitis C," Clinical Gastroenterology and Hepatology, vol. 3, no. 6, pp. 595-603, 2005.

[119] L. Wen, Y. Ma, D. P. Bogdanos et al., "Pédiatrie autoimmune liver diseases: the molecular basis of humoral and cellular immunity," Current Molecular Medicine, vol. 1, no. 3, pp. 379-389, 2001.

[120] M. S. Longhi, Y. Ma, D. P. Bogdanos, P. Cheeseman, G. Mieli-Vergani, and D. Vergani, "Impairment of CD4 $4^{+} \mathrm{CD} 25^{+}$ regulatory T-cells in autoimmune liver disease," Journal of Hepatology, vol. 41, no. 1, pp. 31-37, 2004. 
[121] M. S. Longhi, Y. Ma, R. R. Mitry et al., "Effect of CD4 ${ }^{+} C D 25^{+}$ regulatory T-cells on CD8 T-cell function in patients with autoimmune hepatitis," Journal of Autoimmunity, vol. 25, no. 1, pp. 63-71, 2005.

[122] D. P. Bogdanos, M. Lenzi, M. Okamoto et al., "Multiple viral/self immunological cross-reactivity in liver kidney microsomal antibody positive hepatitis C virus-infected patients is associated with the possession of HLA B51," International Journal of Immunopathology and Pharmacology, vol. 17, no. 1, pp. 83-92, 2004.

[123] D. Vergani, D. P. Bogdanos, and H. Baum, "Unusual suspects in primary biliary cirrhosis," Hepatology, vol. 39, no. 1, pp. 38-41, 2004.

[124] D. P. Bogdanos, A. Pares, H. Baum et al., "Disease-specific cross-reactivity between mimicking peptides of heat shock protein of mycobacterium gordonae and dominant epitope of E2 subunit of pyruvate dehydrogenase is common in Spanish but not British patients with primary biliary cirrhosis," Journal of Autoimmunity, vol. 22, no. 4, pp. 353-362, 2004.

[125] D. P. Bogdanos, K. Choudhuri, and D. Vergani, "Molecular mimicry and autoimmune liver disease: virtuous intentions, malign consequences," Liver, vol. 21, no. 4, pp. 225-232, 2001.

[126] L. Vilagut, J. Vila, O. Vinas et al., "Cross-reactivity of anti-Mycobacterium gordonae antibodies with the major mitochondrial autoantigens in primary biliary cirrhosis," Journal of Hepatology, vol. 21, no. 4, pp. 673-677, 1994.

[127] L. Vilagut, A. Parés, O. Viñas, J. Vila, M. T. Jiménez De Anta, and J. Rodés, "Antibodies to mycobacterial $65-\mathrm{kD}$ heat shock protein cross-react with the main mitochondrial antigens in patients with primary biliary cirrhosis," European Journal of Clinical Investigation, vol. 27, no. 8, pp. 667-672, 1997.

[128] S. C. Robson, R. J. Hift, and R. E. Kirsch, "Primary biliary cirrhosis. A retrospective survey at Groote Schuur Hospital, Cape Town," South African Medical Journal, vol. 78, no. 1, pp. 19-22, 1990.

[129] L. Nesher, K. Riesenberg, L. Saidel-Odes, F. Schlaeffer, and R. Smolyakov, "Tuberculosis in African refugees from the Eastern Sub-Sahara region," The Israel Medical Association Journal, vol. 14, pp. 111-114, 2012.

[130] A. K. S. Samanta, A. G. Bhagwat, and M. Mukherjee, "Primary biliary cirrhosis in India," Gut, vol. 14, no. 6, pp. 448-450, 1973.

[131] S. K. Sarin, R. Monga, B. S. Sandhu, B. C. Sharma, P. Sakhuja, and V. Malhotra, "Primary biliary cirrhosis in India," Hepatobiliary and Pancreatic Diseases International, vol. 5, no. 1, pp. 105-109, 2006.

[132] H. Liu, Y. Liu, L. Wang et al., "Prevalence of primary biliary cirrhosis in adults referring hospital for annual health checkup in Southern China," BMC Gastroenterology, vol. 10, p. 100, 2010.

[133] G. M. Hirschfield and P. Invernizzi, "Progress in the genetics of primary biliary cirrhosis," Seminars in Liver Disease, vol. 31, no. 2, pp. 147-156, 2011.

[134] P. Invernizzi, "Human leukocyte antigen in primary biliary cirrhosis: an old story now reviving," Hepatology, vol. 54, no. 2, pp. 714-723, 2011.

[135] P. Invernizzi, M. Ransom, S. Raychaudhuri et al., "Classical HLA-DRB1 and DPB1 alleles account for HLA associations with primary biliary cirrhosis," Genes and Immunity, vol. 13, no. 6, pp. 461-468, 2012.

[136] P. Invernizzi, C. Selmi, F. Poli et al., "Human leukocyte antigen polymorphisms in Italian primary biliary cirrhosis: a multicenter study of 664 patients and 1992 healthy controls," Hepatology, vol. 48, no. 6, pp. 1906-1912, 2008.

[137] G. F. Mells, J. A. B. Floyd, K. I. Morley et al., "Genomewide association study identifies 12 new susceptibility loci for primary biliary cirrhosis," Nature Genetics, vol. 43, no. 4, pp. 332-333, 2011.

[138] C. Selmi, N. J. Torok, A. Affronti, and M. E. Gershwin, "Genomic variants associated with primary biliary cirrhosis," Genome Medicine, vol. 2, no. 1, p. 5, 2010.

[139] A. Tanaka, P. Invernizzi, H. Ohira et al., "Replicated association of $17 \mathrm{q} 12-21$ with susceptibility of primary biliary cirrhosis in a Japanese cohort," Tissue Antigens, vol. 78, no. 1, pp. 65-68, 2011.

[140] A. Tanaka, H. Ohira, K. Kikuchi et al., "Genetic association of Fc receptor-like 3 polymorphisms with susceptibility to primary biliary cirrhosis: ethnic comparative study in Japanese and Italian patients," Tissue Antigens, vol. 77, no. 3, pp. 239-243, 2011.

[141] E. Png, B. Alisjahbana, E. Sahiratmadja et al., "A genome wide association study of pulmonary tuberculosis susceptibility in Indonesians," BMC Medical Genetics, vol. 13, p. 5, 2012.

[142] T. Thye, F. O. Vannberg, S. H. Wong et al., "Genomewide association analyses identifies a susceptibility locus for tuberculosis on chromosome 18q11.2," Nature Genetics, vol. 42, no. 9, pp. 739-741, 2010.

[143] J. L. Casanova and L. Abel, "Genetic dissection of immunity to mycobacteria: the human model," Annual Review of Immunology, vol. 20, pp. 581-620, 2002.

[144] R. Van Crevel, I. Parwati, E. Sahiratmadja et al., "Infection with Mycobacterium tuberculosis Beijing genotype strains is associated with polymorphisms in SLC11A1/NRAMP1 in Indonesian patients with tuberculosis," Journal of Infectious Diseases, vol. 200, no. 11, pp. 1671-1674, 2009.

[145] O. Sela, A. El-Roeiy, and D. A. Isenberg, "A common antiDNA idiotype in sera of patients with active pulmonary tuberculosis," Arthritis and Rheumatism, vol. 30, no. 1, pp. 50-56, 1987.

[146] J. A. Cayla and A. Orcau, "Control of tuberculosis in large cities in developed countries: an organizational problem," BMC Medicine, vol. 9, p. 127, 2011. 


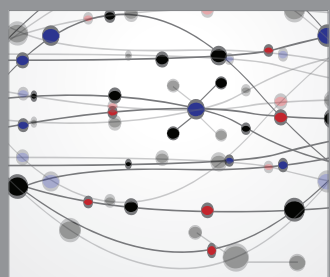

The Scientific World Journal
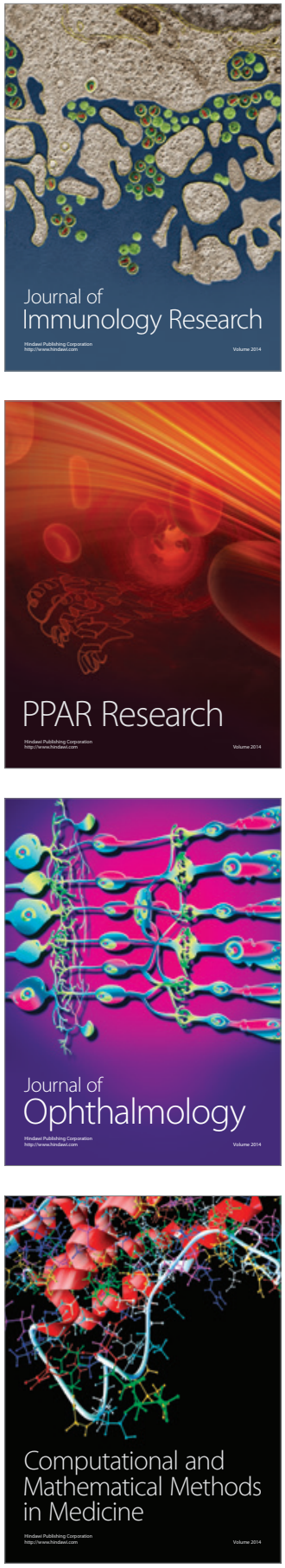

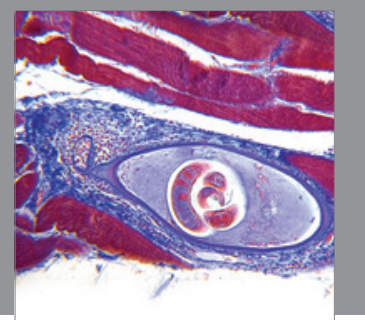

Gastroenterology

Research and Practice
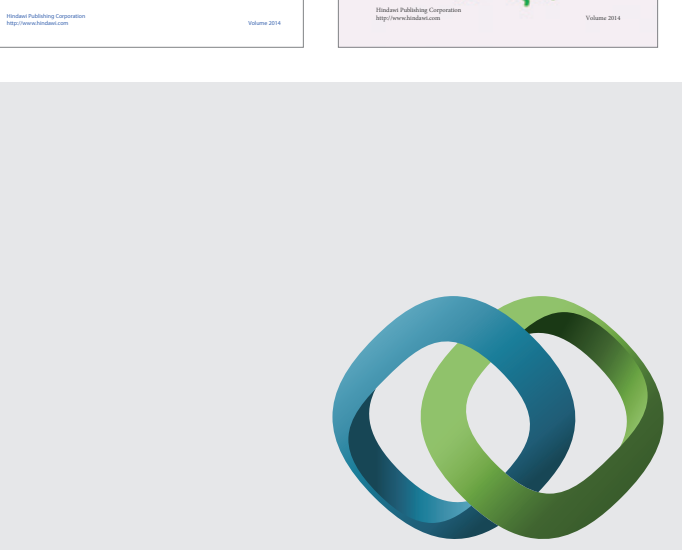

\section{Hindawi}

Submit your manuscripts at

http://www.hindawi.com
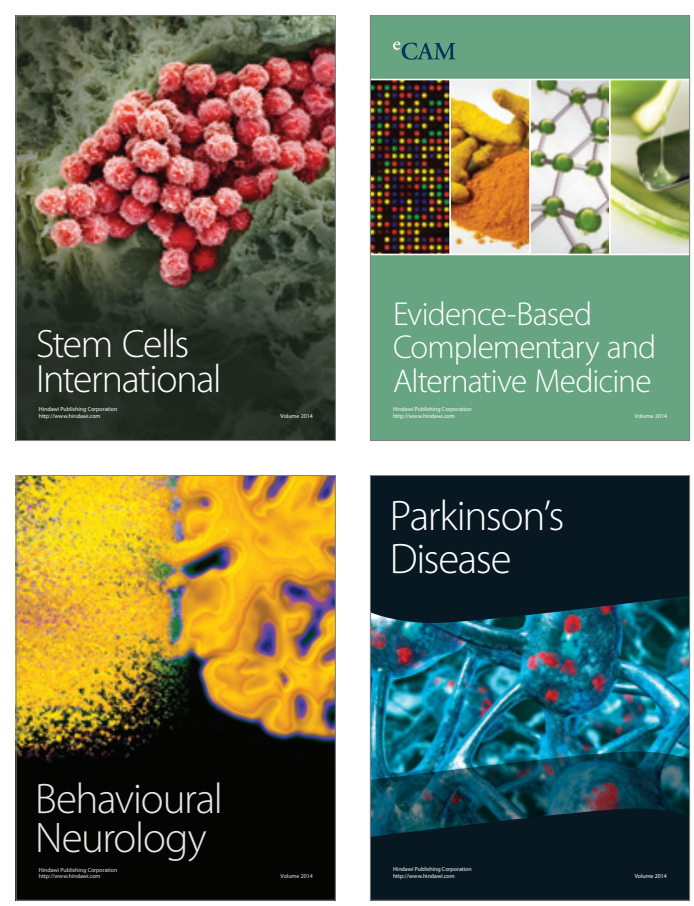

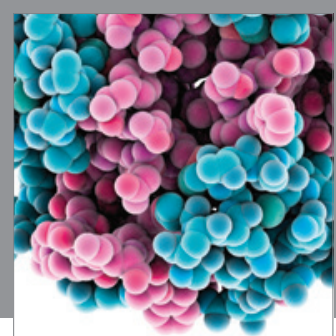

Journal of
Diabetes Research

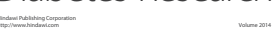

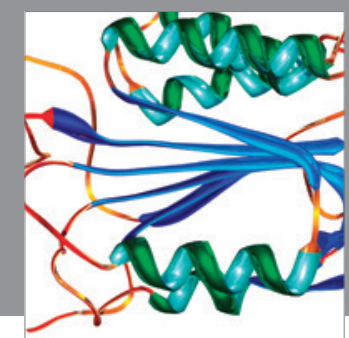

Disease Markers
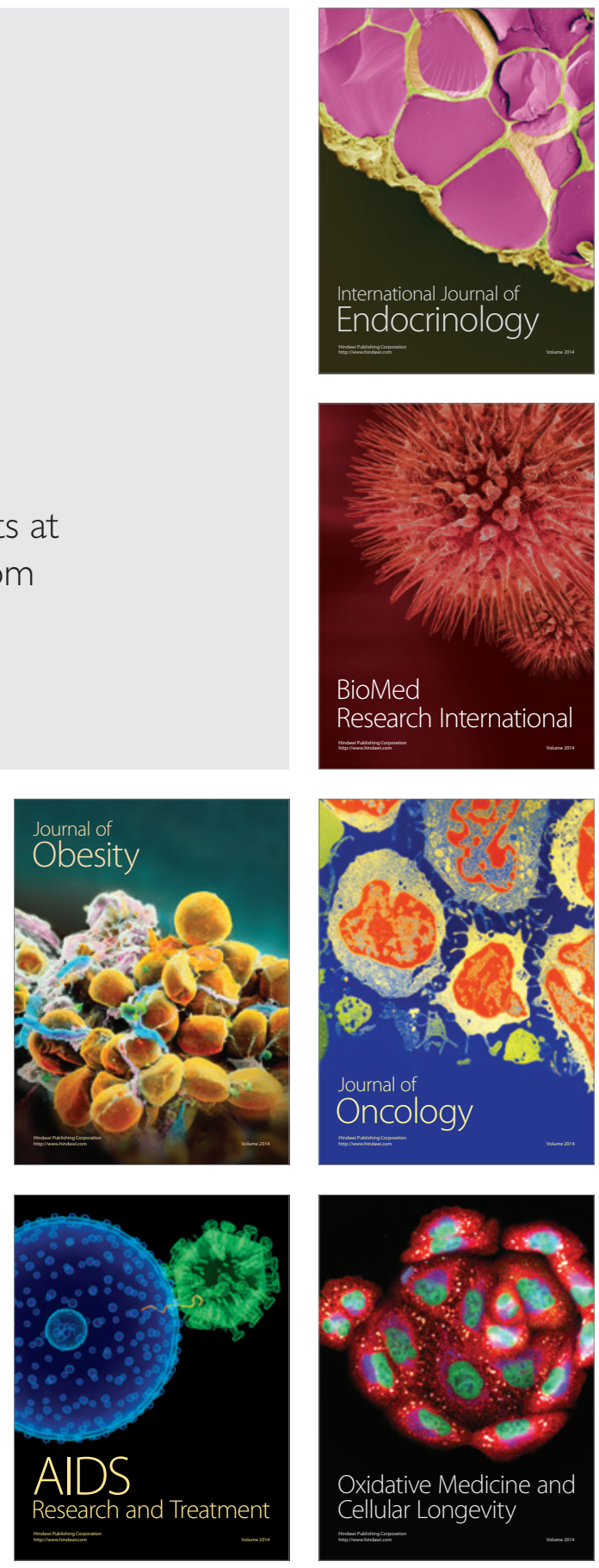